\title{
Specificity and sensitivity of glaucoma detection in the Japanese population using scanning laser polarimetry
}

\section{Shigeo Funaki, Motohiro Shirakashi, Kiyoshi Yaoeda, Haruki Abe, Shiho Kunimatsu, Yasuyuki Suzuki, Goji Tomita, Makoto Araie, Noriko Yamada, Hideya Uchida, Tetsuya Yamamoto, Yoshiaki Kitazawa}

Br J Ophthalmol 2002;86:70-74

See end of article for authors' affiliations

Correspondence to: Shigeo Funaki, MD, Department of

Ophthalmology, Niigata University School of

Medicine, 1-757

Asahimachi-dori, Niigata

951-8510, Japan;

shigeo@muse.ocn.ne.jp

Accepted for publication 17 July 2001
Aims: To investigate the usefulness of the scanning laser polarimeter (GDx; GDx Nerve Fiber Analyzer) for glaucoma detection in the Japanese population, and to investigate the difference in the thickness of retinal nerve fibre layer (RNFL) between normal tension glaucoma (NTG) and primary open angle glaucoma (POAG).

Methods: 69 eyes of 69 normal subjects and 115 eyes of 115 chronic open angle glaucoma patients (60 NTG and 55 POAG patients) were studied. The thickness of RNFL was measured with GDx. An eye was diagnosed as glaucomatous, if at least one original GDx variable showed $p<5 \%$. The difference in thickness of RNFL between the NTG and POAG groups was then investigated.

Results: 46 normal eyes (66.7\%) were diagnosed as not glaucomatous (no variables showing $p<5 \%$ ), and 93 glaucomatous eyes (46 NTG and 47 POAG eyes) (80.9\%) were diagnosed as glaucomatous. Actual values of average thickness, ellipse average, superior average, and superior integral were significantly lower in the POAG group than those in the NTG group.

Conclusions: New variables which elucidate focal RNFL defects or early changes are needed to improve the moderate detection ability found in this present study. The pattern of the change in RNFL may differ in NTG and POAG groups.
A vailability of the scanning laser polarimeter now permits objective and quantitative measurement of the thickness of the retinal nerve fibre layer (RNFL) in vivo. ${ }^{1-10} \mathrm{In}$ normal subjects, measurements of the thickness of RNFL with this instrument resemble those obtained by histological examination. Measurements of the peripapillary thickness of the RNFL in the superior and inferior quadrants were higher than those in the temporal and nasal quadrants. ${ }^{2410-12}$ The thickness of the RNFL has been shown to decrease as the distance from the margin of the optic disc increases. ${ }^{21-13}$ Tjion-Fo-Sang and Lemij ${ }^{14}$ calculated the mean retardation in six selected areas of $16 \times 16$ squares of 256 pixels from each of four quadrants (superior, inferior, temporal, and nasal) and calculated ratios relative to the nasal segment for the superior and inferior quadrants using scanning laser polarimetry. In their study, they found that scanning laser polarimetry had a sensitivity of $96 \%$ and a specificity of $93 \%$ for detecting glaucoma. Based on their report, ${ }^{14}$ a method of examining subsets of the 65536 pixels $(256 \times 256$ picture elements $)$ was implemented into the new software of the scanning laser polarimeter (Nerve Fiber Analyzer GDx (GDx), Laser Diagnostic Technologies, San Diego, CA, USA), ${ }^{15}$ and this GDx does indeed aid in glaucoma diagnosis. Weinreb et $a l^{15}$ reported that examining three variables of GDx (average thickness, ellipse modulation, and average ellipse thickness) and combining them in a linear discriminant function in their study population resulted in sensitivity and specificity of $74 \%$ and $92 \%$, respectively. In the present study, we investigated the usefulness of the GDx for glaucoma diagnosis in the Japanese population. A normative database of GDx has been generated from each race and generation. A p value for each GDx variable was obtained from the software by comparing the current RNFL data with their normative database. Race differences in thickness of the RNFL have been described in previous reports. ${ }^{10}$ The proportion of myopic refraction is greater in Japanese than in white populations. ${ }^{16}$ The present study consists of Japanese normal subjects and glaucoma patients, and sheds light on the usefulness of GDx software for glaucoma detection in Asia. Optic nerve head appearance and pattern of visual field changes may differ between patients with normal tension glaucoma (NTG) and those with primary open angle glaucoma (POAG). ${ }^{17-21}$ The difference in the pattern of RNFL change in Japanese patients with high and low tension glaucoma has been reported. ${ }^{22}$ We previously reported that the relation between values for RNFL thickness obtained by scanning laser polarimetry and the visual field indices were different in NTG and POAG eyes. ${ }^{23}{ }^{24}$ We also studied the difference in the sensitivity of GDx variables between the NTG and POAG groups in the present study.

\section{SUBJECTS AND METHODS}

A total of 69 normal eyes of 69 Japanese volunteers ( 33 males and 36 females), and 115 eyes of 115 chronic open angle glaucoma patients (63 males and 52 females) (60 NTG patients and 55 POAG patients) were studied. Normal volunteers and glaucoma patients were recruited from Niigata University, University of Tokyo, and Gifu University. Normal subjects ranged in age from 22 to 71 years, mean 40.9 (SD 14.8) years, and glaucoma patients ranged in age from 26 to 81 years, mean 58.3 (12.0) years. Informed consent was obtained from each normal subject and glaucoma patient. Normal subjects and glaucoma patients showed normal appearance of the cornea. The eyes of normal subjects studied had a best corrected visual acuity above $20 / 25$, a refractive error (spherical equivalent) between -5 and +5 dioptres (average $=-1.33$ (SD 1.57) dioptres), a normal intraocular pressure $\leqslant 21 \mathrm{~mm} \mathrm{Hg}$, normal appearance of the optic disc, no significant ocular disease found upon routine ophthalmological examination, and normal visual field with reliable measurements (fixation loss $<20 \%$, false negative and false positive $<15 \%$ ) performed on the central 24-2 or 30-2 program of the Humphrey field analyser (HFA) (Allergan-Humphrey, San Leandro, CA, USA). Results of the glaucoma hemifield test did not show outside 
Table 1 The average of mean deviation of Humphrey field analysis in each stage of the two glaucoma groups

\begin{tabular}{llll}
\hline Stage & NTG group & POAG group & p Value \\
\hline Early stage & $-2.42(1.54)(n=22)$ & $-2.59(1.39)(n=21)$ & 0.706 \\
Moderately progressed stage & $-9.42(2.82)(n=23)$ & $-10.02(3.14)(n=17)$ & 0.530 \\
Progressed stage & $-20.51(4.72)(n=15)$ & $-23.61(4.88)(n=17)$ & 0.079 \\
Total & $-9.62(7.65)(n=60)$ & $-11.38(9.40)(n=55)$ & 0.271 \\
\hline
\end{tabular}

NTG = normal tension glaucoma, POAG = primary open angle glaucoma

Comparisons between groups were done using Student's $t$ test. Data are reported as mean (SD).

normal limits in each eye. $p$ Values for mean deviation and pattern standard deviation were not $<0.05$. All chronic open angle glaucoma patients had the following characteristics: normal open angle; typical glaucomatous optic disc cupping and visual field defects; no history of retrobulbar optic neuritis, anterior ischaemic optic neuropathy, or intracranial lesion. The intraocular pressure of NTG patients without therapy was below $21 \mathrm{~mm} \mathrm{Hg}$ on repeated measurements, whereas pretreatment intraocular pressure of POAG patients was 21 mm Hg or above. ${ }^{24}$ Patients who had a refractive error (spherical equivalent) that exceeded -5 dioptres (D) were excluded from the study (patients' average refractive error was -1.07 (2.04) dioptres, with no significant difference in the refractive error between the normal subjects and chronic open angle glaucoma patients). The eyes of glaucoma patients studied had a best corrected visual acuity above 20/25. HFA (central 30-2 program) was performed on each patient. With reliable measurements of visual field (fixation loss $<20 \%$, false negative and false positive $<15 \%$ ), the evaluation of glaucomatous visual field defects was made based on a liberal criteria $(\geqslant 2$ adjacent points of $\geqslant 5 \mathrm{~dB}$ loss each, $\geqslant 1$ adjacent point of $\geqslant 10$ $\mathrm{dB}$ loss each, or difference of $\geqslant 5 \mathrm{~dB}$ across nasal horizontal meridian at $\geqslant 2$ adjacent points) of a previous report. ${ }^{25}$ In the present study, the glaucoma patients were divided into the three groups by results obtained from the HFA central 30-2 program as follows: 43 eyes (NTG:POAG $=22: 21$ ) in the early stage of the disease (mean deviation $(\mathrm{MD}) \geqslant-5 \mathrm{~d} \mathrm{~B}$ ), 40 eyes $(\mathrm{NTG}: \mathrm{POAG}=23: 17)$ in the moderately progressed stage $(-5$ $\mathrm{dB}>\mathrm{MD}>-15 \mathrm{~dB}$ ), and 32 eyes (NTG:POAG $=15: 17)$ in the progressed stage $(\mathrm{MD} \leqslant-15 \mathrm{~dB})$. The average $(\mathrm{SD})$ value of the $\mathrm{MD}$ in all glaucoma patients was $-10.47(8.54) \mathrm{dB}$. The average (SD) MD value for each stage of the two groups is shown in Table 1 . The average (SD) MD in NTG and POAG patients was $-9.62(7.65) \mathrm{dB}$ and -11.38 (9.40) dB, respectively, with no significant difference $(\mathrm{p}=0.27 \mathrm{l}$, Student's $t$ test). The average (SD) age in NTG and POAG patients was 60.0 (10.3) years and 56.6 (13.6) years, respectively, showing no significant difference $(\mathrm{p}=0.137$, Student's $t$ test). The average (SD) refractive error in NTG and POAG patients was -1.19 (2.04) dioptres and -0.94 (2.04) dioptres, respectively, also with no significant difference $(\mathrm{p}=$ 0.513 , Student's $t$ test).

The thickness of the RNFL was measured with a scanning laser polarimeter. Its basic principles and technical characteristics have been described extensively. ${ }^{1-3}$ Briefly, this instrument consists of a confocal scanning laser ophthalmoscope with a polarisation modulator, a cornea polarisation compensator, and a polarisation detection unit. Measurement of RNFL thickness is based on the assumption that the RNFL possesses birefringent properties that change (retard) the state of polarisation of an illuminating laser beam. This change can be measured by determining the phase shift between extraordinary and ordinary beams. The extent of retardation is linearly correlated with the thickness of the RNFL. Approximately one degree of retardation corresponds to $7.4 \mu \mathrm{m}$ of RNFL thickness as measured histologically after removal of the cornea. ${ }^{1}$ The light source of the instrument consists of a near infrared diode laser (wavelength $780 \mathrm{~nm}$ ) in which the state of polarisation has been modulated. The polarised light penetrates the birefringent RNFL and is partially reflected from the deeper layer of the retina. It is separated from the illuminating light beam by a non-polarising beam splitter. The state of polarisation of the light is analysed by the polarisation detection unit. Then the electrical signal from the detector is digitised and stored in the memory of a personal computer for later analysis. A total of 65536 retinal locations $(256 \times 256$ pixels $)$ are tested, allowing the examiner to create a retardation map in which the thickness of the RNFL is measured for each retinal location.

In the present study, a 15 degree field size was used and the optic disc was centred in the middle of the image for all image acquisitions. The pupils were not dilated. All measurements were obtained from a mean of three images. The average SD of RNFL thickness in retinal locations (pixels) in the mean of the three images was within $8 \mu \mathrm{m}$ in each eye. The 10 pixel wide band was located concentric with the optic disc margin at 1.75 disc diameters. The margin of the optic disc was approximated by an ellipse placed around the inner margin of the peripapillary scleral ring. To calculate absolute values in the integral of RNFL thickness, errors in magnification were corrected using Bengtsson's correction ${ }^{26}$ with axial length. All analyses at one location were performed by the same operator. Before starting this study, we confirmed that interoperator variation was negligible.

The GDx variables examined in the present study were symmetry, superior ratio, inferior ratio, superior/nasal, max modulation, ellipse modulation, ellipse average, superior average, inferior average, average thickness, and superior integral (Table 2). GDx number was excluded from the study because it is not indicated with the probability. A total of 1500 pixels per quadrant ( 120 degrees for superior and inferior quadrants, 50 degrees for the temporal quadrant, and 70 degrees for the nasal quadrant) peripheral to an ellipse 1.75 disc diameters from the centre of the disc was used to calculate symmetry, superior ratio, inferior ratio, superior/nasal, and max modulation. Ellipse modulation, ellipse average, superior average, and inferior average were calculated using pixels within the 10 pixel-wide elliptical band that was automatically positioned concentric with the disc margin outline and 1.75 disc diameters from the centre of the optic disc.

When at least one GDx variable out of 11 variables described above had a $\mathrm{p}$ value $<5 \%$, we defined that eye as glaucomatous by GDx. We studied the specificity and sensitivity of glaucoma diagnosis in Japanese normal subjects and glaucoma patients. We also studied the difference in sensitivity between the NTG and POAG group. Comparisons between groups were done using Student's $t$ test and multivariate logistic regression analysis. The thickness of the RNFL may be changed related to not only the visual field change but also age. $^{24710}$ The relation between visual field change and refractive error has been reported. ${ }^{27}$ In the present study, we selected multivariate logistic regression analysis to analyse these complicated correlating factors. Comparisons between the OAG group and normal subjects were done using multivariate logistic regression analysis ( $p$ value calculated by using the OAG group and normal subjects as dependent variables, and 
Table 2 The GDx variables used in the present study

\begin{tabular}{|c|c|}
\hline Superior ratio: & $\begin{array}{l}\text { the ratio of the average } 1500 \text { thickest pixels in the superior quadrant divided by the average of the } 1500 \text { thickest pixels in the } \\
\text { inferior quadrant. } \\
\text { the ratio of the average of the } 1500 \text { thickest pixels in the superior quadrant divided by the average of the } 1500 \text { median pixels in the } \\
\text { temporal quadrant. }\end{array}$ \\
\hline Inferior ratio: & $\begin{array}{l}\text { the ratio of the average of the } 1500 \text { thickest pixels in the inferior quadrant divided by the average of the } 1500 \text { median pixels in the } \\
\text { temporal quadrant. }\end{array}$ \\
\hline Superior/nasal: & $\begin{array}{l}\text { the average of the } 1500 \text { thickest pixels in the superior quadrant divided by the average of the } 1500 \text { median pixels in the nasal } \\
\text { quadrant. }\end{array}$ \\
\hline Average thickness: & the average of all pixels outside the disc margin. \\
\hline Max modulation: & $\begin{array}{l}\text { first, the average of the } 1500 \text { thickest points in the superior and inferior quadrants was calculated, then the average of } 1500 \text { median } \\
\text { points in the nasal and temporal quadrants were calculated and the lowest of these four values was subtracted from the highest, then } \\
\text { that value divided by the lowest value. }\end{array}$ \\
\hline Ellipse modulation: & $\begin{array}{l}\text { thinnest pixel within the elliptical band was subtracted from the thickest pixels within the elliptical band, then the total divided by the } \\
\text { value of the thinnest pixel. }\end{array}$ \\
\hline $\begin{array}{l}\text { Ellipse average: } \\
\text { Superior average: } \\
\text { Inferior average: } \\
\text { Superior integral: }\end{array}$ & $\begin{array}{l}\text { average thickness of the pixels within the elliptical band surrounding the optic nerve. } \\
\text { the average thickness of the pixels within the elliptical band in the superior quadrant. } \\
\text { the average thickness of the pixels within the elliptical band in the inferior quadrant } \\
\text { the total area under the curve and within the superior portion of the elliptical band surrounding the optic nerve. }\end{array}$ \\
\hline
\end{tabular}

age, refractive error, individual variable GDx $\mathrm{p}<5 \%$ or not as independent variables, and $\mathrm{p}$ value calculated by using the OAG group and normal subjects as dependent variables, and age, refractive error, actual value of a GDx variable as independent variables). Also comparisons between NTG and POAG groups were done using multivariate logistic regression analysis ( $p$ value calculated by using the NTG and POAG group as dependent variables, and age, refractive error, MD, individual variable GDx $p<5 \%$ or not as independent variables, and $p$ value calculated by using the NTG and POAG group as dependent variables, and age, refractive error, MD, actual value of a GDx variable as independent variables). Trend by severity was tested by Cochran-Armitage's test. A level of $p<0.05$ was accepted as statistically significant. Data were reported as mean (SD).

\section{RESULTS}

Forty six of 69 normal eyes $(66.7 \%)$ were diagnosed as not glaucomatous by GDx. Ninety three of 115 glaucomatous eyes $(80.9 \%)$ were diagnosed as glaucomatous by GDx. The number of GDx variables with $\mathrm{p}<5 \%$ in the 23 normal eyes diagnosed as glaucomatous by GDx were from 1 to 4, mean 2.0 (SD 1.1). Superior/nasal showed lowest specificity and highest sensitivity (Tables 3 and 4).
Table 3 shows the sensitivity and specificity of each GDx variable in all glaucomatous eyes and normal subjects. It also shows the average of each GDx variable within each group, as well as the difference between the glaucoma group and normal subjects (multivariate logistic regression analysis). The specificity of each GDx variable was symmetry: $90.0 \%$, superior ratio: $92.8 \%$, inferior ratio: $92.8 \%$, superior/nasal: $87.0 \%$ max modulation: $91.3 \%$, ellipse modulation: $91.3 \%$, average thickness: $98.6 \%$, ellipse average: $97.1 \%$, superior average: $95.7 \%$, inferior average: $98.6 \%$, and superior integral: $100 \%$. In average thickness, ellipse average, superior average, inferior average, and superior integral, there was no significant difference between the OAG group and normal subjects in $\mathrm{p}<5 \%$ or not (multivariate logistic regression analysis). Actual values of symmetry, average thickness, ellipse average, superior average, and superior integral showed no significant difference between normal subjects and the glaucoma group (multivariate logistic regression analysis).

Table 4 shows the sensitivity of each GDx variable in the NTG and the POAG groups. The sensitivity of superior/nasal, max modulation, ellipse modulation, and superior average was significantly lower in the NTG group than in the POAG group (multivariate logistic regression analysis). Table 3 also shows the average of each GDx variable within each group, as well as the difference between the two groups (multivariate

Table 3 Sensitivity and specificity of GDx variables in all glaucomatous eyes and normal subjects, average of each GDx variable in each group, and the difference between the chronic open angle glaucoma group and normal subjects

\begin{tabular}{lllllll}
\hline & Sensitivity & Specificity & $\begin{array}{l}\text { Average value in } \\
\text { patients with OAG }\end{array}$ & $\begin{array}{l}\text { Average value in } \\
\text { normal subjects }\end{array}$ & $\mathrm{p}^{*}$ & $\mathrm{p}^{* *}$ \\
\hline S & $29.6 \%$ & $90.0 \%$ & $1.08(0.22)$ & $1.07(0.16)$ & 0.018 & 0.729 \\
SR & $29.6 \%$ & $92.8 \%$ & $1.69(0.38)$ & $2.33(0.68)$ & 0.003 & $<0.001$ \\
IR & $35.7 \%$ & $92.8 \%$ & $1.61(0.38)$ & $2.17(0.54)$ & $<0.001$ & $<0.001$ \\
S/N & $56.5 \%$ & $87.0 \%$ & $1.49(0.26)$ & $1.87(0.32)$ & $<0.001$ & $<0.001$ \\
MM & $45.2 \%$ & $91.3 \%$ & $0.92(0.76)$ & $1.45(0.60)$ & $<0.001$ & 0.010 \\
EM & $44.3 \%$ & $91.3 \%$ & $1.39(0.61)$ & $2.14(0.74)$ & 0.002 & $<0.001$ \\
AT & $0.9 \%$ & $98.6 \%$ & $68.6(13.0)$ & $72.0(14.4)$ & 0.720 & 0.711 \\
EA & $3.5 \%$ & $97.1 \%$ & $69.2(13.7)$ & $74.4(14.4)$ & 0.946 & 0.363 \\
SA & $9.6 \%$ & $95.7 \%$ & $76.3(17.5)$ & $84.3(19.0)$ & 0.246 & 0.057 \\
IA & $11.3 \%$ & $98.6 \%$ & $73.8(16.3)$ & $80.9(18.4)$ & 0.060 & 0.047 \\
SI & $3.5 \%$ & $100 \%$ & $0.24(0.09)$ & $0.25(0.06)$ & 0.768 & 0.883 \\
\hline
\end{tabular}

$\mathrm{S}=$ symmetry, $\mathrm{SR}=$ superior ratio, $\mathrm{IR}=$ inferior ratio, $\mathrm{S} / \mathrm{N}=$ superior/nasal, $\mathrm{MM}=$ max modulation, $\mathrm{EM}=$ ellipse modulation, $A T=$ average thickness, $E A=$ ellipse average, $S A=$ superior average, $I A=$ inferior average, $\mathrm{SI}=$ superior integral, $\mathrm{OAG}=$ normal tension glaucoma and primary open angle glaucoma. Comparisons between groups were done using multivariate logistic regression analysis ( $p$ value $\left(p^{*}\right)$ calculated by using the OAG group and normal subjects as dependent variables, and age, refractive error individual variable GDx $p<5 \%$ or not as independent variables (11 ways), and $p$ value $\left(p^{* *}\right)$ calculated by using the OAG group and normal subjects as dependent variables, and age, refractive error, actual value of a GDx variable as independent variables $(11$ ways)). Data are reported as mean (SD). 
Table 4 Specificity, sensitivity of GDx variables in normal tension glaucoma and primary open angle glaucoma, and the difference between the two groups. Average of each GDx variable in each group and the difference between the two groups

\begin{tabular}{llllllll}
\hline & Specificity & $\begin{array}{c}\text { Sensitivity } \\
\text { in NTG }\end{array}$ & $\begin{array}{c}\text { Sensitivity } \\
\text { in POAG }\end{array}$ & $\begin{array}{l}\text { Average value } \\
\text { in NTG patients }\end{array}$ & $\begin{array}{l}\text { Average value in } \\
\text { POAG patients }\end{array}$ & $\mathrm{p}^{*}$ & $\mathrm{p}^{* *}$ \\
\hline S & $90.0 \%$ & $33.3 \%$ & $25.5 \%$ & $1.11(0.23)$ & $1.05(0.21)$ & 0.348 & 0.075 \\
SR & $92.8 \%$ & $21.7 \%$ & $38.2 \%$ & $1.72(0.34)$ & $1.66(0.41)$ & 0.067 & 0.183 \\
IR & $92.8 \%$ & $30.0 \%$ & $41.8 \%$ & $1.59(0.31)$ & $1.63(0.45)$ & 0.214 & 0.588 \\
S/N & $87.0 \%$ & $48.3 \%$ & $67.3 \%$ & $1.54(0.26)$ & $1.45(0.27)$ & 0.043 & 0.075 \\
MM & $91.3 \%$ & $35.0 \%$ & $56.4 \%$ & $0.99(0.98)$ & $0.85(0.40)$ & 0.031 & 0.322 \\
EM & $91.3 \%$ & $35.0 \%$ & $54.5 \%$ & $1.39(0.49)$ & $1.39(0.72)$ & 0.027 & 0.679 \\
AT & $98.6 \%$ & $0 \%$ & $1.8 \%$ & $71.3(13.5)$ & $65.6(11.8)$ & 0.755 & 0.005 \\
EA & $97.1 \%$ & $1.7 \%$ & $5.5 \%$ & $71.5(14.3)$ & $66.7(12.7)$ & 0.126 & 0.018 \\
SA & $95.7 \%$ & $3.3 \%$ & $16.4 \%$ & $80.1(17.7)$ & $72.2(16.6)$ & 0.018 & 0.002 \\
IA & $98.6 \%$ & $6.7 \%$ & $16.4 \%$ & $75.7(17.0)$ & $71.8(15.4)$ & 0.139 & 0.111 \\
SI & $100 \%$ & $3.3 \%$ & $3.6 \%$ & $0.26(0.11)$ & $0.22(0.06)$ & 0.691 & 0.001
\end{tabular}

$S=$ symmetry, $S R=$ superior ratio, $I R=$ inferior ratio, $S / N=$ superior/nasal, $M M=$ max modulation, $E M=$ ellipse modulation, $\mathrm{AT}=$ average thickness, $\mathrm{EA}=$ ellipse average, $\mathrm{SA}=$ superior average, $\mathrm{IA}=$ inferior average, $\mathrm{SI}=$ superior integral, NTG = normal tension glaucoma, POAG = primary open angle glaucoma. Comparisons between groups were done using multivariate logistic regression analysis ( $p$ value $\left(p^{*}\right)$ calculated by using the NTG and POAG group as dependent variables, and age, refractive error, MD, individual variable GDx $p<5 \%$ or not as independent variables (11 ways), and $p$ value $\left(p^{* *}\right)$ calculated by using the NTG and POAG group as dependent variables, and age, refractive error, MD, actual value of a GDx variable as independent variables (11 ways)). Data are reported as mean (SD).

Table 5 Sensitivity using GDx at each stage of all glaucomatous eyes and the two glaucoma groups

\begin{tabular}{llll}
\hline Stage & All glaucomatous eyes & NTG group & POAG group \\
\hline Early stage & $29 / 43(67.4 \%)$ & $14 / 22(63.6 \%)$ & $15 / 21(71.4 \%)$ \\
Moderately progressed stage & $32 / 40(80.0 \%)$ & $17 / 23(73.9 \%)$ & $15 / 17(88.2 \%)$ \\
Progressed stage & $32 / 32(100 \%)$ & $15 / 15(100 \%)$ & $17 / 17(100 \%)$ \\
Total & $93 / 115(80.9 \%)$ & $46 / 60(76.7 \%)$ & $47 / 55(85.5 \%)$ \\
\hline
\end{tabular}

NTG = normal tension glaucoma, POAG = primary open angle glaucoma .

logistic regression analysis). The values of average thickness, ellipse average, superior average, and superior integral were significantly lower in the POAG group than those in the NTG group.

Sensitivity for each stage of the two groups is shown in Table 5. Sensitivity of all glaucomatous eyes in early, moderately progressed, and progressed stages was $67.4 \%$, $80.0 \%$, and $100 \%$, respectively with sensitivity increasing gradually with progressives stages of the disease (CochranArmitage's test; $\mathrm{p}<0.001$ ).

\section{DISCUSSION}

In the present study, GDx proved moderately useful for glaucoma detection with an overall specificity of $66.7 \%$ and sensitivity of $80.9 \%$. Because glaucomatous eyes in the present study had abnormal appearing optic discs and because abnormal appearing discs are correlated with abnormal RNFL, sensitivities might be artificially high in the present study. Actual values of symmetry, average thickness, ellipse average, superior average, and superior integral did not show a significant difference between normal subjects and the glaucoma group (multivariate logistic regression analysis). However, the overlap in RNFL thickness between normal eyes and glaucomatous eyes has been reported. ${ }^{2}$ There is also wide variation among normal individuals in the thickness of the RNFL as measured by scanning laser polarimetry, ${ }^{246}{ }^{10}$ which may be due to differences in prenatal regression of retinal ganglion cells in individuals. ${ }^{28}{ }^{29}$ In the present study, this wide variation might be the cause of the lack of significant difference between normal subjects and glaucoma patients in five of the GDx variables. Weinreb et $a l^{15}$ reported that using three variables of GDx (average thickness, ellipse modulation, and average ellipse thickness) resulted in sensitivity and specificity of $74 \%$ and $92 \%$, respectively. The difference between the study of Weinreb et $\mathrm{al}^{15}$ and the present study may be because the methods of calculating sensitivity and specificity were different. Although the studies concerned with glaucoma diagnosis using scanning laser polarimeter have been reported, ${ }^{14}{ }^{15}$ 30-32 this is the first trial to investigate the usefulness of each original GDx variable. We arbitrarily defined the parameters for diagnosis of glaucoma in the present study, choosing the simplest application of GDx which would be most practical in non-specialty clinics. In the present study, GDx variables using an absolute value showed lower sensitivity than those using a ratio, and this was also consistent with the study of Kubota et $a l^{22}$ which was in Japanese glaucoma patients. These results may be due to the wide distribution of RNFL thickness data generated by the GDx for which the linear relation between corneal polarisation axis and posterior segment retardation parameters is partly responsible. ${ }^{33}$ In average thickness, ellipse average, superior average, inferior average, and superior integral there was no significant difference in $\mathrm{p}<5 \%$ between the OAG group and normal subjects in the present study. Moreover, sensitivity of average thickness and ellipse average was $0.9 \%$ and $3.5 \%$, respectively. Although this may be due to the overlap in RNFL thickness between normal and glaucomatous eyes, ${ }^{2}$ the difference in the usefulness of these two parameters in the study of Weinreb et $a l^{15}$ and the present study is still unclear. Difference due to race and the percentage of NTG patients might have influenced these results.

We previously reported that the relation between values for RNFL thickness obtained by scanning laser polarimetry and the visual field indices was different in NTG and POAG eyes. $^{23}{ }^{24}$ In the present study, the sensitivity of superior/nasal, max modulation, ellipse modulation, and superior average was significantly lower in the NTG group than in the POAG group. Also, the actual values of average thickness, ellipse 
average, superior average, and superior integral were significantly lower in the POAG group than in the NTG group. High tension glaucomatous eyes had significantly more diffuse visual field damage than NTG eyes. ${ }^{18}$ The present result might indicate that diffuse RNFL loss in the POAG eyes was more easily detected in GDx, because the minimum unit calculated in most GDx variables is a quadrant and this may cause the software to fail to reflect a focal RNFL defect. Others reported that there actually is a difference in visual field defects between normal tension glaucoma and high tension glaucoma $^{2021}$; previous reports and the present study might indicate the difference in RNFL and the different pathogenesis between the two groups. Because the number of patients diagnosed as being NTG might vary depending on race, there may be inherent error in glaucoma detection by GDx.

The GDx software has been tested in many clinical trials. GDx variables are calculated by two methods-using a total of 1500 pixels per quadrant peripheral to an ellipse of 1.75 disc diameters or using pixels within the 10 pixel-wide elliptical band that is automatically positioned concentric with the disc margin outline and 1.75 disc diameters from the centre of the optic disc. Although the thickness of the RNFL is overestimated by scanning laser polarimetry when there is parapapillary chorioretinal atrophy, ${ }^{2}$ the average of the 1500 median pixels in the temporal quadrant has been used as the representative value of the temporal quadrant to avoid this artefact. Also, distinct quadrant divisions (120 degrees for superior and inferior, 50 degrees for temporal, and 70 degrees for nasal quadrant) are used. However, there are still some problems - for example, if the optic disc size is large, the total of 1500 pixels per quadrant peripheral to the ellipse 1.75 disc diameters will be calculated from a smaller pixel population. We previously reported that the integral of RNFL thickness obtained by scanning laser polarimetry depended on the size of the optic disc. ${ }^{34}$ These problems concerning optic disc size must be considered. NFA-GDx can assist in the diagnosis of glaucoma; however, new variables which elucidate focal or early changes in the RNFL are needed. Although GDx variables are currently calculated from four divided regions more detailed division may be needed. We hope that the results obtained in the present study will advance the detection of glaucoma.

\section{ACKNOWLEDGMENTS}

The authors have no proprietary interest in any of the equipment or materials used in this study.

\section{Authors' affiliations}

S Funaki, M Shirakashi, K Yaoeda, H Abe, Department of Ophthalmology, Niigata University School of Medicine, Niigata, Japan

S Kunimatsu, Y Suzuki, G Tomita, M Araie, Department of

Ophthalmology, University of Tokyo, Tokyo, Japan

N Yamada, H Uchida, T Yamamoto, Y Kitazawa, Department of

Ophthalmology, Gifu University, Gifu, Japan

\section{REFERENCES}

1 Weinreb RN, Dreher AW, Coleman A, et al. Histopathologic validation of Fourier-ellipsometry measurements of retinal nerve fiber layer thickness. Arch Ophthalmol 1990;108:557-60.

2 Weinreb RN, Shakiba S, Zangwill L. Scanning laser polarimetry to measure the nerve fiber layer of normal and glaucomatous eyes. Am J Ophthalmol 1995;119:627-36

3 Weinreb RN, Shakiba S, Sample PA, et al. Association between quantitative nerve fiber layer measurement and visual field loss in glaucoma. Am J Ophthalmol 1995;120:732-8.

4 Chi Q-M, Tomita G, Inazumi K, et al. Evaluation of the effect of aging on the retinal nerve fiber layer thickness using scanning laser polarimetry. J Glaucoma 1995;4:406-13.
5 Shirakashi $M$ Abe H, Sawaguchi S. Relationship between retinal nerve fiber layer thickness and visual field loss in glaucoma. Atarashii Ganka (Journal of the Eye) 1996;13:597-9.

6 Niessen AGJE, van den Berg TJTP, Langerhorst CT, et al. Retinal nerve fiber layer assessment by scanning laser polarimetry and standardized photography. Am J Ophthalmol 1996;121:484-93.

7 Tjon-Fo-Sang MJ, de Vries J, Lemij HG. Measurement by nerve fiber analyzer of retinal nerve fiber layer thickness in normal subjects and patients with ocular hypertension. Am J Ophthalmol 1996;122:220-7.

8 Junghardt A, Schmid MK, Schipper I, et al. Reproducibility of the data determined by scanning laser polarimetry. Graefes Arch Clin Exp Ophthalmol 1996;234:628-32.

9 Anton A, Zangwill L, Emdadi A, et al. Nerve fiber layer measurements with scanning laser polarimetry in ocular hypertension. Arch Ophthalmol $1997 ; 115: 331-4$

10 Poinoosawmy D, Fontana L, Wu JX, et al. Variation of nerve fibre layer thickness measurements with age and ethnicity by scanning laser polarimetry. Br J Ophthalmol 1997;81:350-4.

11 Radius RL. Thickness of the retinal nerve fiber layer in primate eyes. Arch Ophthalmol 1980;98:1625-9.

12 Quigley HA, Addicks EM. Quantitative studies of retinal nerve fiber layer defects. Arch Ophthalmol 1982;100:807-14.

13 Varma R, Skaf M, Barron E. Retinal nerve fiber layer thickness in normal human eyes. Ophthalmology 1996;103:2114-19.

14 Tijion-Fo-Sang MJ, Lemii HG. The sensitivity and specificity of nerve fiber layer measurements in glaucoma as determined with scanning laser polarimetry. Am J Ophthalmol 1997;123:62-9.

15 Weinreb RN, Zangwill L, Berry CC, et al. Detection of glaucoma with scanning laser polarimetry. Arch Ophthalmol 1998;116:1583-9.

16 Shiose Y, Kitazawa Y, Tsukahara S, et al. Epidemiology of glaucoma in Japan: a nationwide glaucoma survey. Jpn J Ophthalmol 1991;35:133-55

17 Yamagami J, Araie M, Shirato S. A comparative study of optic nerve head in low- and high-tension glaucomas. Graefes Arch Clin Exp Ophthalmol 1992;230:446-50.

18 Araie M, Yamagami J, Suzuki Y. Visual field defects in normal-tension and high-tension glaucoma. Ophthalmology 1993;100:1808-14.

19 Drance SM. The concept of chronic open-angle glaucoma: a personal review. Ophthalmologica 1996;210:251-6

20 Caprioli J, Spaeth GL. Comparison of visual field defects in the low-tension glaucomas with those in the high-tension glaucomas. Am J Ophthalmol 1984;97:730-7.

21 King D, Drance SM, Douglas G, et al. Comparison of visual field defects in normal-tension glaucoma and high-tension glaucoma. Am J Ophthalmol 1986;101:204-7.

22 Kubota T, Khalil AK, Honda M, et al. Comparative study of retinal nerve fiber layer damage in Japanese patients with normal- and high-tension glaucoma. J Glaucoma 1999;8:363-6.

23 Shirakashi $\mathbf{M}$, Funaki S, Funaki $\mathrm{H}$, et al. Measurement of retinal nerve fibre layer by scanning laser polarimetry and high pass resolution perimetry in normal tension glaucoma with relatively high or low intraocular pressure. Br J Ophthalmol 1999;83:353-7.

24 Shirakashi $\mathbf{M}$, Abe H, Sawaguchi S, et al. Measurement of thickness of retinal nerve fiber layer by scanning laser polarimetry and high-pass resolution perimetry in patients with primary open-angle or normal-tension glaucoma. Acta Ophthalmol Scand 1997;75:641-4.

25 Caprioli J. Automated perimetry in glaucoma. Am J Ophthalmol $1991 ; 111: 235-9$.

26 Bengtsson B, Krakau CE. Correction of optic disc measurements on fundus photographs. Graefes Arch Clin Exp Ophthalmol 1992;230:24-8.

27 Araie $M$, Arai $M$, Koseki $N$, et al. Influence of myopic refraction on visual field defects in normal tension and primary open angle glaucoma. Jpn J Ophthalmol 1995;39:60-4.

28 Rakic $\mathbf{P}$, Riley KP. Overproduction and elimination of retinal axons in the fetal rhesus monkey. Science 1983;219:1441-4

29 Provis JM, van Driel D, Billson FA, et al. Human fetal optic nerve: overproduction and elimination of retinal axons during development. $J$ Comp Neurol 1985;238:92-100.

30 Yamada N, Chen PP, Mills RP, et al. Glaucoma screening using the scanning laser polarimeter. J Glaucoma 2000;9:254-61.

31 Trible JR, Schultz RO, Robinson JC, et al. Accuracy of scanning laser polarimetry in the diagnosis of glaucoma. Arch Ophthalmol 1999; 117:1298-304

32 Horn FK, Jonas JB, Martus $\mathrm{P}$, et al. Polarimetric measurement of retina nerve fiber layer thickness in glaucoma diagnosis. J Glaucoma 1999;8:353-62.

33 Greenfield DS, Knighton RW, Huang XR. Effect of corneal polarization axis on assessment of retinal nerve fiber layer thickness by scanning lase polarimetry. Am J Ophthalmol 2000;129:715-22.

34 Funaki S, Shirakashi M, Abe H. Relation between size of optic disc and thickness of retinal nerve fibre layer in normal subjects. $\mathrm{Br} J$ Ophthalmol 1998;82:1242-5. 